\title{
XMM-Newton X-ray and HST weak gravitational lensing study of the extremely X-ray luminous galaxy cluster Cl J120958.9+495352 ( $z=0.902)$
}

\author{
Sophia Thölken ${ }^{1}$, Tim Schrabback ${ }^{1}$, Thomas H. Reiprich ${ }^{1}$, Lorenzo Lovisari ${ }^{2}$, Steven W. Allen ${ }^{3,4,5}$, Henk Hoekstra ${ }^{6}$, \\ Douglas Applegate ${ }^{7}$, Axel Buddendiek ${ }^{1}$, and Amalia Hicks ${ }^{8}$ \\ 1 Argelander-Institut für Astronomie, Universität Bonn, Auf dem Hügel 71, 53121 Bonn, Germany \\ e-mail: thoelken@astro.uni-bonn.de \\ 2 Harvard-Smithsonian Center for Astrophysics, 60 Garden Street, Cambridge, MA 02138, USA \\ 3 Kavli Institute for Particle Astrophysics and Cosmology, Stanford University, 452 Lomita Mall, Stanford, CA 94305-4085, USA \\ 4 Department of Physics, Stanford University, 452 Lomita Mall, Stanford, CA 94305-4085, USA \\ 5 SLAC National Accelerator Laboratory, 2575 Sand Hill Road, Menlo Park, CA 94025, USA \\ ${ }^{6}$ Leiden Observatory, Leiden University, PO Box 9513, 2300 RA Leiden, The Netherlands \\ 7 Kavli Institute for Cosmological Physics, University of Chicago, 5640 S Ellis Ave, Chicago, IL 60637, USA \\ 8 Cadmus, Energy Services Division, 16 N. Carroll Street, Suite 900, Madison, WI 53703, USA
}

Received 1 April 2017 / Accepted 18 August 2017

\begin{abstract}
Context. Observations of relaxed, massive, and distant clusters can provide important tests of standard cosmological models, for example by using the gas mass fraction. To perform this test, the dynamical state of the cluster and its gas properties have to be investigated. X-ray analyses provide one of the best opportunities to access this information and to determine important properties such as temperature profiles, gas mass, and the total X-ray hydrostatic mass. For the last of these, weak gravitational lensing analyses are complementary independent probes that are essential in order to test whether X-ray masses could be biased.

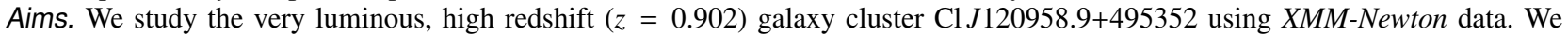
measure global cluster properties and study the temperature profile and the cooling time to investigate the dynamical status with respect to the presence of a cool core. We use Hubble Space Telescope (HST) weak lensing data to estimate its total mass and determine the gas mass fraction.

Methods. We perform a spectral analysis using an XMM-Newton observation of $15 \mathrm{ks}$ cleaned exposure time. As the treatment of the background is crucial, we use two different approaches to account for the background emission to verify our results. We account for point spread function effects and deproject our results to estimate the gas mass fraction of the cluster. We measure weak lensing galaxy shapes from mosaic HST imaging and select background galaxies photometrically in combination with imaging data from the William Herschel Telescope.

Results. The X-ray luminosity of $\mathrm{Cl} J 120958.9+495352$ in the $0.1-2.4 \mathrm{keV}$ band estimated from our XMM-Newton data is $L_{\mathrm{X}}=$ $\left(13.4_{-1.0}^{+1.2}\right) \times 10^{44} \mathrm{erg} / \mathrm{s}$ and thus it is one of the most X-ray luminous clusters known at similarly high redshift. We find clear indications for the presence of a cool core from the temperature profile and the central cooling time, which is very rare at such high redshifts. Based on the weak lensing analysis, we estimate a cluster mass of $M_{500} / 10^{14} M_{\odot}=4.4_{-2.0}^{+2.2}$ (stat.) \pm 0.6 (sys.) and a gas mass fraction of $f_{\text {gas }, 2500}=0.11_{-0.03}^{+0.06}$ in good agreement with previous findings for high redshift and local clusters.
\end{abstract}

Key words. galaxies: clusters: general - galaxies: clusters: individual: Cl J120958.9+495352 - X-rays: galaxies: clusters gravitational lensing: weak

\section{Introduction}

In the paradigm of hierarchical structure formation very massive and distant clusters should be extremely rare. These clusters provide the opportunity for many interesting astrophysical and cosmological studies. The gas mass fraction $\left(f_{\text {gas }}\right)$ of dynamically relaxed clusters is an important probe of cosmological models (Allen et al. 2008; Mantz et al. 2014) as the matter content of these objects should approximately match the matter content of the universe (e.g., White et al. 1993; Allen et al. 2011, and references therein). In particular high-redshift clusters are of interest where the leverage on the cosmology is largest.

The cooling time for these clusters is very short and the presence of a cool core is believed to be strongly related to the dynamical status of the cluster (e.g., Hudson et al. 2010). McDonald et al. (2017) studied the evolution of the ICM and cool core clusters over the past $10 \mathrm{Gyr}$. Their results imply that from redshift $z=0$ to $z=1.2$ cool cores basically do not evolve in size, density, and mass. Additionally, the level of agreement of the properties of these rare clusters with existing scaling relations (e.g., Reichert et al. 2011; Pratt et al. 2009) has great significance for cosmology as the properties can provide tests of these scaling laws and assess whether they are in line with standard cosmological predictions.

So far, only a few of these rare, relaxed, massive, and high redshift objects have been found; two examples are $\mathrm{Cl} J 0046.3+8530$ (Maughan et al. 2004b) and Cl J1226.9+3332 (Maughan et al. 2004a). In the Massive Cluster Survey (MACS; 
Ebeling et al. 2007, 2010), many interesting objects have been identified, for example extreme cooling in cluster cores such as MACS J1931.8-2634 (Ehlert et al. 2011), and a number of dynamically relaxed clusters that can be used for cosmological tests. However, almost all of those relaxed clusters are at smaller redshifts than the object studied here. Two of the most distant clusters at $z>1, \mathrm{Cl} J 1415.1+3612(z=1.028)$ and 3C $186(z=1.067)$, were studied in detail by Babyk (2014) and Siemiginowska et al. (2010) using deep Chandra observations. The observations revealed a cool core for both objects with a short cooling time for $\mathrm{Cl} J 1415.1+3612$ within the core region of $<0.2 \mathrm{Gyr}$ and a gas-mass fraction consistent with local clusters for 3C 186 . With respect to the luminosity, another extreme example is the El Gordo galaxy cluster at $z=0.87$ with $L_{X}=(2.19 \pm 0.11) \times 10^{45} h_{70}^{-2} \mathrm{erg} / \mathrm{s}$ (Menanteau et al. 2012) which is one of the most massive and luminous clusters found so far.

For cosmological tests, the total cluster mass is an important quantity for which weak gravitational lensing provides an independent probe in addition to the X-ray hydrostatic mass. The gravitational potential imprints coherent distortions onto the observed shapes of background galaxies (e.g., Bartelmann \& Schneider 2001; Schneider 2006). Measurements of these weak lensing distortions directly constrain the projected mass distributions and cluster masses (Hoekstra et al. 2013). These measurements are sensitive to the total matter distribution, including both dark matter and baryons. Especially at high redshifts, the Hubble Space Telescope (HST) is an essential tool for the analysis of such objects as ground-based telescopes are not able to resolve the shapes of the very distant background galaxies.

Recently, Buddendiek et al. (2015) performed a combined search of distant massive clusters using ROSAT All Sky Survey and Sloan Digital Sky Survey (SDSS) data covering an area of $10000 \mathrm{deg}^{2}$. They found 83 high-grade candidates for X-ray luminous clusters between $0.6<z<1$ and obtained William Herschel Telescope (WHT) or Large Binocular Telescope (LBT) imaging to confirm the candidates. One of the clusters they found is special in many respects: $\mathrm{Cl} J 120958.9+495352$ is the most X-ray luminous cluster in their sample. Also, it has the second highest spectroscopically confirmed redshift in their sample, and their richness and Sunyaev-Zel'dovich (SZ) measurements independently indicate a high cluster mass. According to the Planck catalog of SZ sources (Planck Collaboration XVI 2015)

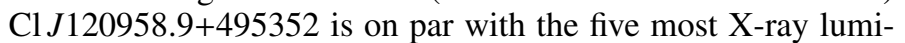
nous clusters found at $z \sim 0.9$. It is thus a valuable candidate for a distant cooling-core cluster and provides a great opportunity to study one of these rare systems in detail.

In this work we perform a spectroscopic XMM-Newton and HST weak lensing study of this extraordinary object found by Buddendiek et al. (2015). We investigate the temperature profile with respect to the presence of a cool core and determine the cooling time within $<100 \mathrm{kpc}$. In Sect. 2 we describe the properties of $\mathrm{Cl} J 120958.9+495352$, the data reduction procedure, and the analysis strategy for HST and XMM-Newton and for the $X M M-N e w t o n$ background. Section 3 gives the results, which are then discussed in Sect. 4.

Throughout the analysis we use a flat $\Lambda$ cold dark matter (CDM) cosmology with $H_{0}=70 \mathrm{~km} \mathrm{~s}^{-1} \mathrm{Mpc}^{-1}, \Omega_{\mathrm{m}}=0.3$, and $\Omega_{\Lambda}=0.7$. All uncertainties are given at the $68 \%$ confidence level and overdensities refer to the critical density. All magnitudes are in the $\mathrm{AB}$ system.
Table 1. Details of the XMM-Newton observation of Cl J120958.9+495352.

\begin{tabular}{cccclc}
\hline \hline Rev. & Date & RA & Dec & $\begin{array}{l}\text { Cleaned } \\
\text { exp. time }\end{array}$ & Filter \\
\hline 2545 & Oct. 2013 & 182.512 & 49.926 & $9.6 \mathrm{ks}$ & thick \\
2546 & Nov. 2013 & 182.510 & 49.924 & $5.1 \mathrm{ks}$ & thick \\
\hline
\end{tabular}

\section{Observations and data analysis}

\subsection{XMM-Newton analysis}

\subsubsection{Data reduction}

$\mathrm{Cl} J 120958.9+495352$ is the most luminous cluster in the sample of Buddendiek et al. (2015). From the ROSAT data, this cluster already appears to be one of the most luminous known at high redshifts with an observer-frame luminosity of $L_{\mathrm{obs}, 0.1-2.4 \mathrm{keV}=}=$ $20.3 \pm 6.2 \times 10^{44} \mathrm{erg} / \mathrm{s}$ (Buddendiek et al. 2015). They measure the spectroscopic redshift to be $z=0.902$ and their SZ data yields a mass of $M_{500}=(5.3 \pm 1.5) \times 10^{14} h_{70}^{-1} M_{\odot}$.

We analyze XMM-Newton observations of the cluster with $\sim 15$ ks cleaned exposure time (XMM-Newton observation IDs 0722530101 and 0722530201 , PI of the joint XMM-Newton and HST program: T. Schrabback). The observations were performed in October and November 2013 (see Table 1) and were executed over the course of two revolutions, which we analyze simultaneously.

Following the standard data reduction procedure ${ }^{1}$ using SAS version 14.0.0, we use the ODF data and apply cifbuild to catch up with the latest calibration and odfingest to update the ODF summary file with the necessary instrumental housekeeping information. Then we proceed by applying emchain and epchain (for MOS and PN detector, respectively) to create calibrated event files.

On these calibrated files we apply the following filters for the event pattern of the triggered CCD pixels (the numbering is based to the ASCA GRADE selection) and the quality flag of the pixels: PATTERN $\leq 12$ for the MOS detectors, for PN PATTERN $=0 ;$ FLAG $=0$ for both detectors. Because of anomalous features on CCD4 of MOS1, we additionally filter out events falling onto this chip. The CCD3 and CCD6 of MOS1 were damaged by micro meteorite events and the data of these detectors cannot be used.

In a next step we create light curves for both revolutions and all detectors in the energy range $0.3-10 \mathrm{keV}$. The observation in the second revolution shows strong flaring for a large fraction of the exposure time. We apply a $3 \sigma$ clipping to all the light curves to filter the flared time intervals and inspected the light curves afterwards which then show no further hint of flaring. This removes approximately half of the exposure time for the second observation (revolution 2546).

To detect point sources in the field of view (FOV), we create images from the event files for all detectors in five energy bands between $0.2-12 \mathrm{keV}$. These images are provided in the task edetect_chain.

\subsubsection{Spectral fitting}

An X-ray image of the cluster is shown in Fig. 1. We select three annular regions around the center and choose the region sizes such that we can achieve a $\mathrm{S} / \mathrm{Bkg}$ ratio (i.e.,

1 See heasarc.gsfc.nasa.gov/docs/xmm/abc/ 


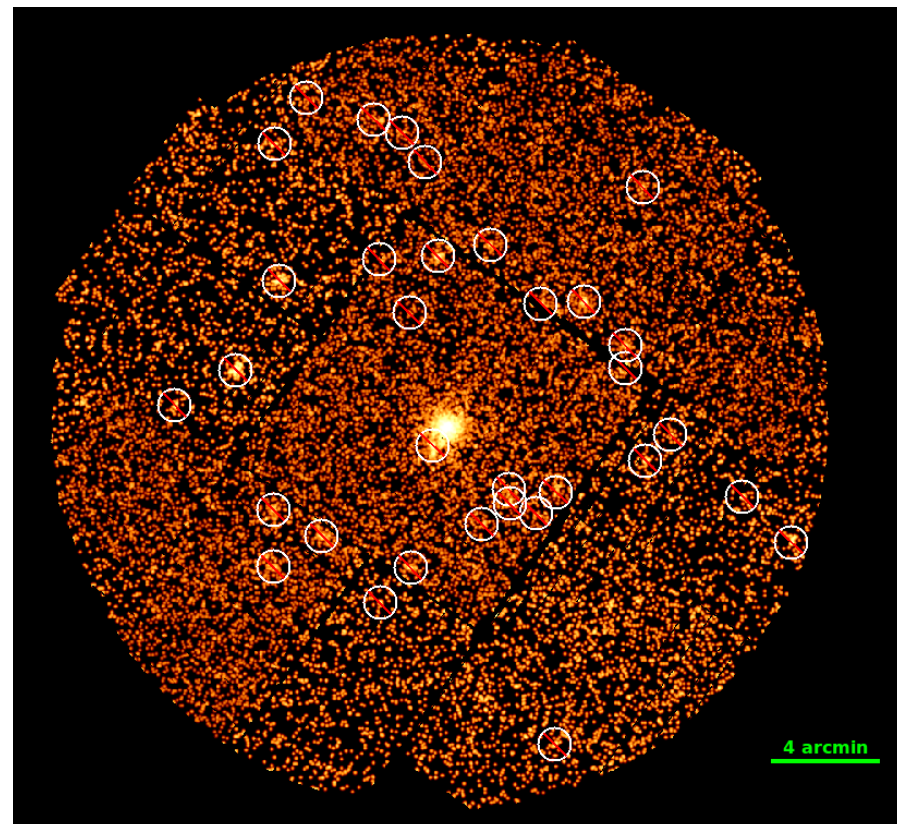

Fig. 1. Combined, cleaned, exposure corrected, and smoothed MOS image of $\mathrm{Cl} J 120958.9+495352$. White circles show the excluded point sources.

counts $_{\text {source }} /$ counts $_{\text {bkg }}$ ) of $\sim 1$ in the outermost annulus and higher for the inner regions to avoid systematic biases. The final regions are $0^{\prime}-0,3,0,3-0,8$, and $0{ }^{\prime} 8-1 ! 3$. We fit the spectra of all annuli and for all detectors and the two observations simultaneously using the Cash-Statistic (cstat option) in XSPEC. For the cluster emission we use an absorbed APEC model with a column density from Willingale et al. (2013), which also includes molecular hydrogen and the solar metal abundance table from Asplund et al. (2009). We assume the same abundance in all annuli and thus link the corresponding model parameters. The $X M M-N e w t o n$ point spread function (PSF) is $\sim 17^{\prime \prime} \mathrm{HEW}$. We correct for the effect of photon mixing between different annuli due of the PSF as described in Sect. 2.1.5.

From our HST data (Sect. 3.1) we estimate $R_{500}=1.8$ and therefore, for the estimation of the global cluster properties, we extract spectra in this region. For the analysis of such a high redshift cluster, the background treatment is crucial. The different background components are described in Sects. 2.1.3 and 2.1.4 and we follow two approaches for the treatment of the background:

1. Background modeling. One approach is to model all the different background components individually in the fitting procedure. These components are described in the following sections. We determine models for the quiescent particle background and the X-ray background and use them in the fitting of the cluster emission. We additionally introduce a power-law model to account for the residual soft proton emission, which is left over emission after the flare filtering. The index is linked for the two MOS detectors while the normalizations for each detector are independent. We use an energy range between $0.7-10 \mathrm{keV}$. The results of this approach can be found in Sect. 3.2.

2. Background subtraction. The cluster has a small extent on the sky, thus we do not expect significant cluster emission beyond $R_{200}=2.7^{\prime}$ estimated from our HST data. For this reason we are able to subtract the full background from the spectra. To do so, we extract background spectra in an annulus between $3^{\prime}-5^{\prime}$. This region lies completely on the MOS

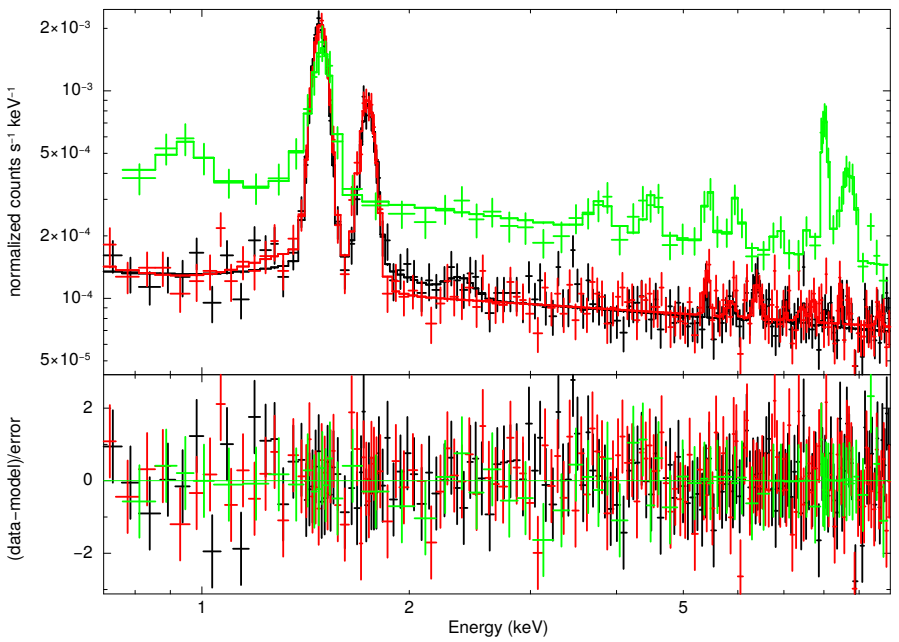

Fig. 2. Spectra and best fit models of the QPB obtained from the filterwheel-closed observations and extracted on the central chip in the region $0^{\prime}-5^{\prime}$ for MOS1 (black), MOS2 (red), and PN (green) and normalized to the extraction area.

CCD1 chips which is important because the particle background shows strong variations between the different chips. Also for PN this region is close enough to the source extraction region to properly model the $\mathrm{Ni}$ and $\mathrm{Cu}$ lines. As for the first method, the energy range is $0.7-10 \mathrm{keV}$ and the results of this procedure are described in Sect. 3.2.

\subsubsection{Quiescent particle background}

The quiescent particle background (QPB) is caused by highly energetic particles interacting with the detector and the surrounding material. It is composed of a continuum emission and fluorescent lines from various elements contained in the assembly of the satellite. XMM-Newton is equipped with a filter wheel system which can be used to measure the level of the QPB. When the filter is closed, only the high energy particles can penetrate the filter and a spectrum of the QPB can be obtained. We use merged event files of the filter-wheel-closed observations which are close to the time of the observation (revolution 2514-2597 for the MOS detectors and 2467-2597 for PN). The continuum part of the spectrum can be described by two power laws, while the fluorescent lines are modeled by Gaussians. The QPB varies for all detectors and with the position on the detector. Therefore, we fit the model in two regions - from $0^{\prime}-5^{\prime}$ (the source region, which lies completely on CCD1 for the MOS destectors) and from $77^{\prime}-12^{\prime}$ (the region where we determine the X-ray background, see Sect. 2.1.4) - for all detectors independently. For the QPB, diagonal responses are used in the fit and no ancillary response file (ARF) is applied as these particles do not suffer from instrumental effects such as vignetting. The spectra with the best fit models are shown in Fig. 2. When fitting the cluster emission, the QPB normalizations of the power-law components and the Gaussian lines are allowed to vary separately by $\pm 20 \%$, due to possible spatial and temporal variations of the QPB.

\subsubsection{X-ray background}

The X-ray background (XRBG) emission is caused by different sources: 1) a local component and solar wind charge exchange; 2) a component from the Milky Way halo plasma; and 3) the superposition of the X-ray emission from distant AGNs causing a 


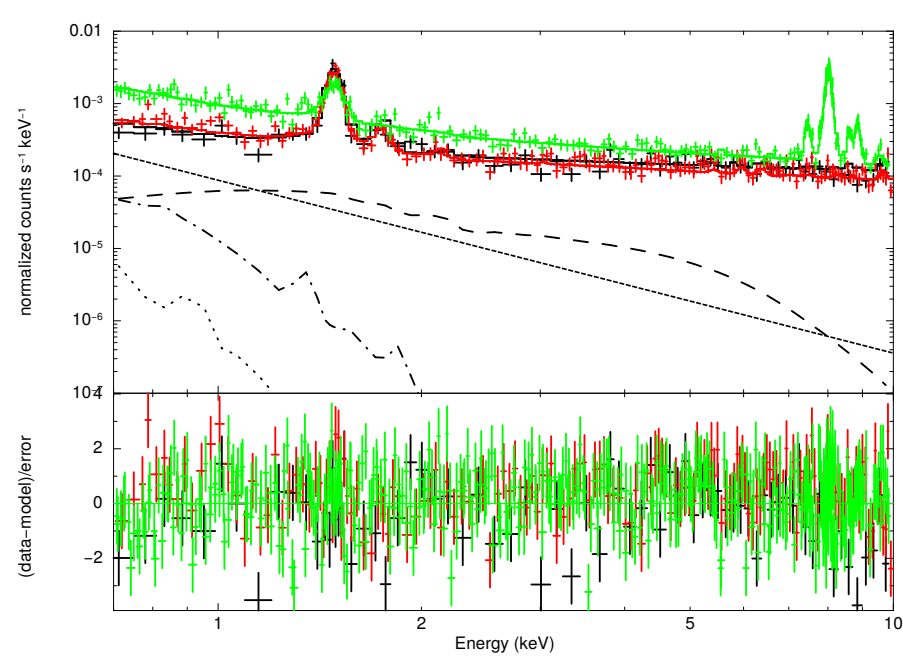

Fig. 3. Spectra and best fit models for the XRBG + QPB for MOS1 (black), MOS2 (red), and PN (green) in the region $7^{\prime}-12^{\prime}$. The different components of the XRBG are shown as dotted, dash-dotted, and dashed lines for the local, halo, and CXB component, respectively. The power law component for the residual soft proton emission is shown as shortdashed line. For the spectra and models of the QPB see Fig. 2.

diffuse background (CXB). To model these background components we extract a spectrum in a region $7^{\prime}-12^{\prime}$, where no cluster emission is expected. Additionally, ROSAT All-Sky-Survey data $^{2}$ are used to support the estimation of the background parameters at energies between $0.1-2.0 \mathrm{keV}$. The first XRBG component can be modeled using an APEC model with temperature and normalization as free fitting parameters. The redshift and the abundance are set to 0 and 1 , respectively. The second component can be described by an absorbed APEC model. The superposition of AGN emission was analyzed by De Luca \& Molendi (2004) and can be modeled by an absorbed power law with a photon index of 1.41. We accounted for the particle background in this annulus by using the previously determined model in Sect. 2.1.3 in the region $7^{\prime}-12^{\prime}$ with two floating multiplicative constants $( \pm 20 \%)$ for the continuum part and the fluorescent lines. We additionally introduce a power-law model to account for the residual soft proton emission. Also for this model we use diagonal response matrices.

The XRBG spectra and the best fit models for the different components are shown in Fig. 3 for the off-axis region between $7^{\prime}$ and $12^{\prime}$.

\subsubsection{PSF correction}

The extent of the cluster on the sky is small; therefore, we have to choose annular region sizes which suffer from the PSF size of XMM-Newton. This causes "mixing" of photons i.e., photons originating from a certain region on the sky are detected in another region on the detector. This has an impact on the spectra and influences the measurements, especially the determination of the temperature profile. To avoid this we introduce a PSF correction. The XMM-Newton task arfgen allows us to calculate cross-region ARFs. Via these cross-region ARFs the effective area for the emission coming from one particular region, but detected in another, is estimated. These ARFs can then be used in the fitting process to account for the PSF effects. Therefore, we introduced additional absorbed APEC models for each

\footnotetext{
2 Obtained with the HEASARC X-ray background tool heasarc.gsfc.nasa.gov/cgi-bin/Tools/xraybg/xraybg.pl
}

combination of photon mixing (e.g., photons from region 1 on the sky but detected in region 2 on the detector, etc.). These models use the cross-region ARFs and the model parameters are linked to the parameters of the annulus the emission truly originates from, as described in the corresponding SAS-thread ${ }^{3}$. We neglect the PSF effects for the emission coming from the outermost annulus to the two inner annuli as the effective area for this mixing is close to zero.

\subsection{HST analysis}

Here we perform a weak gravitational lensing analysis based

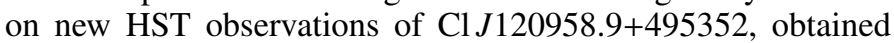
within the joint XMM-Newton+HST program (HST program ID 13493). Weak lensing measurements require accurate measurements of the shapes of background galaxies well behind the cluster. Given the high redshift of $\mathrm{Cl} J 120958.9+495352$, typical weak lensing background galaxies are at redshifts $z \gtrsim 1.4$. As most of them are unresolved in ground-based seeing-limited data, HST observations are key for this study. Specifically, we analyze observations obtained with the Advanced Camera for Surveys (ACS) in the $F 606 W$ filter in a $2 \times 2$ mosaic covering a $\sim 6.5 \times 6$. 6 area (corresponding to $\sim 3.0 \times 3.1 \mathrm{Mpc}^{2}$ ), with integration times of $1.9 \mathrm{ks}$ per pointing, each split into four exposures.

The data reduction and analysis is conducted with the same pipeline that was used for the weak lensing analysis of high-redshift galaxy clusters from the South Pole Telescope Sunyaev Zel'dovich Survey (Bleem et al. 2015) presented in Schrabback et al. (2018, hereafter S16). Therefore, we only summarize the main analysis steps here and refer the reader to S16 for further details.

For the ACS data reduction we employ basic calibrations from CALACS, the correction for charge-transfer inefficiency from Massey et al. (2014), MultiDrizzle (Koekemoer et al. 2003) for the cosmic ray removal and stacking, and scripts for the image registration and improvement of masks from Schrabback et al. (2010).

We detect objects using Source Extractor (Bertin \& Arnouts 1996) and measure shapes using the KSB+ formalism (Kaiser et al. 1995; Luppino \& Kaiser 1997; Hoekstra et al. 1998) as implemented by Erben et al. (2001) with adaptions for HST measurements described in Schrabback et al. (2007, 2010). In particular, we apply a model for the temporally and spatially varying HST PSF constructed from a principal component analysis of ACS stellar field observations. In order to estimate cluster masses from weak lensing, accurate knowledge of the source redshift distribution is required. Here we follow the approach from S16, who first apply a color selection to remove cluster galaxies from the source sample, and then estimate the redshift distribution based on CANDELS photometric redshift catalogs (Skelton et al. 2014), to which they apply consistent selection criteria, as used in the cluster fields, and statistical corrections for photometric redshift outliers.

For the color selection we make use of additional $i$-band observations of $\mathrm{Cl} J 120958.9+495352$ obtained with the Prime Focus Camera PFIP (Prime Focus Imaging Platform) on the $4.2 \mathrm{~m}$ WHT (ID: W14AN004, PI: Hoekstra) on March 26, 2014. These observations were taken with the new red-optimized RED+4 detector, which has an imaging area of $4096 \times 4112$ pixels, with a pixel scale of $00^{\prime \prime} .27$ and an $18^{\prime} \times 18^{\prime}$ field of view. We reduce these data using theli (Erben et al. 2005; Schirmer 2013),

\footnotetext{
cosmos. esa. int/web/xmm-newton/sas-thread-esasspec
} 


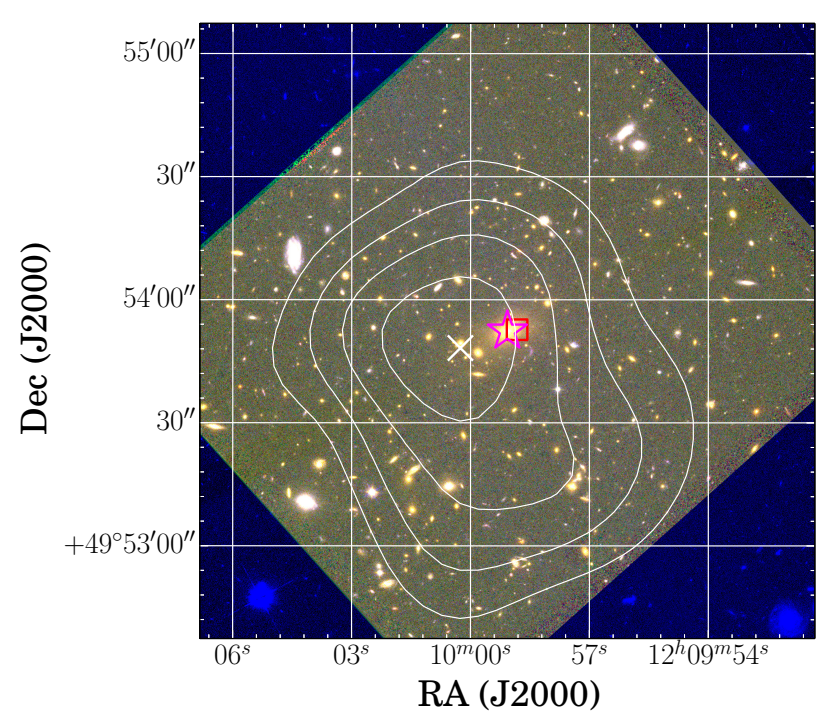

Fig. 4. HST 2'5 $\times 2$ ' 5 color image of $\mathrm{Cl} J 120958.9+495352$ based on the ACS/WFC F606W (blue) and WFC3/IR F105W (green), and F140W (red) imaging. The white contours indicate the signal-to-noise ratio of the weak lensing mass reconstruction, starting at $2 \sigma$ in steps of $0.5 \sigma$ with the cross marking the peak position, which is consistent with the X-ray peak (red square) and BCG position (magenta star) within the uncertainty of $23^{\prime \prime}$ in each direction.

co-adding exposures of a total integration time of $13.5 \mathrm{ks}$ and reaching a $5 \sigma$ limit of $i_{\mathrm{WHT}, \mathrm{lim}} \simeq 25.8$ in circular apertures of $2^{\prime \prime}$, with an image quality of $2 r_{\mathrm{f}}=1^{\prime \prime} .2$, where $r_{\mathrm{f}}$ corresponds to the FLUX_RADIUS parameter from Source Extractor. We use SDSS (SDSS Collaboration et al. 2017) for the photometric calibration and convolve the ACS F606W imaging to the groundbased resolution to measure $V_{606 \text {,con }}-i_{\text {WHT }}$ colors. For galaxies at the cluster redshift the $4000 \AA$ /Balmer break is located within this filter pair. Therefore, by selecting very blue galaxies in this color, we can cleanly remove the cluster galaxies, while selecting the majority of the $z \gtrsim 1.4$ background sources carrying the lensing signal (see S16). To account for the increased scatter at faint magnitudes we apply a magnitude-dependent selection $V_{606, \text { con }}-i_{\mathrm{WHT}}<0.16\left(V_{606, \text { con }}-i_{\mathrm{WHT}}<-0.04\right)$ for galaxies with magnitudes $24<V_{606}<25.5\left(25.5<V_{606}<26\right)$ measured in 0!:7 diameter apertures from the non-convolved ACS images. These cuts correspond to a color selection in the CANDELS catalogs of $V_{606}-I_{814}<0.2\left(V_{606}-I_{814}<0.0\right)$. In order to select consistent galaxy populations between the cluster field and the CANDELS catalogs we additionally apply consistent lensing shape cuts and add photometric scatter to the deeper CANDELS catalogs as empirically estimated in S16. The depth

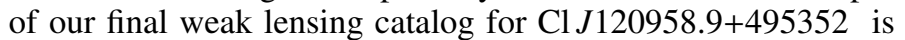
mostly limited by the mediocre seeing conditions during the WHT observations, which require us to substantially degrade the $F 606 \mathrm{~W}$ images in the PSF matching for the color measurements. As a result, we have to apply a rather stringent selection $V_{606 \text {,auto }}<25.8$ based on the Source Extractor auto magnitude, which results in a final galaxy number density of 9.6/ $\operatorname{arcmin}^{2}$, while the shape catalog extends to $V_{606 \text {,auto }} \simeq 26.5$. We therefore recommend that future programs following a similar observing strategy should ensure that complementary ground-based observations are conducted under good seeing conditions in order to fully exploit the statistical power of the HST weak lensing shape catalogs.

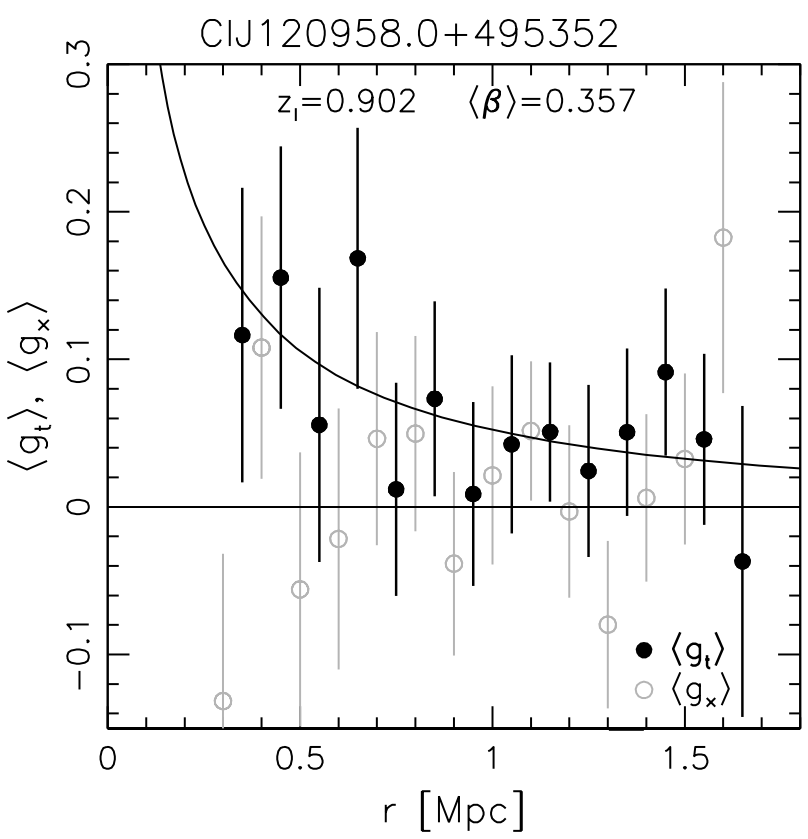

Fig. 5. Tangential reduced shear profile (black solid circles) of Cl J120958.9+495352, measured around the X-ray peak. Here we combine the profiles of four magnitude bins between $24<V_{606 \text {,aper }}<26$, as done in S16. The curve shows the corresponding best fitting NFW model prediction constrained by fitting the data within the range $300 \mathrm{kpc} \leq r \leq 1.5 \mathrm{Mpc}$, assuming the mass-concentration relation from Diemer \& Kravtsov (2015). The gray open circles indicate the reduced cross-shear component, which has been rotated by 45 degrees and constitutes a test for systematics. These points have been shifted by $\mathrm{d} r=-0.05 \mathrm{Mpc}$ for clarity.

Taking the magnitude distribution and shape weights of our color-selected source catalog into account, we estimate an effective mean geometric lensing efficiency of $\langle\beta\rangle=0.357 \pm 0.009$ (sys.) \pm 0.025 (stat.) based on the CANDELS analysis (see S16 for details).

\section{Results}

\subsection{HST results}

In Fig. 4 we show contours of the weak lensing mass reconstruction of $\mathrm{Cl} J 120958.9+495352$, overlaid onto a color image from the ACS/WFC F606W imaging and WFC3/IR imaging obtained in $F 105 W(1.2 \mathrm{ks})$ and $F 140 W(0.8 \mathrm{ks})$. The reconstruction employs a Wiener filter (McInnes et al. 2009; Simon et al. 2009), as further detailed in S16. Divided by the r.m.s. image of the reconstructions of 500 noise fields, the contours indicate the signal-to-noise ratio of the weak lensing mass reconstruction, starting at $2 \sigma$ in steps of $0.5 \sigma$. The reconstruction peaks at $\mathrm{RA}=12: 10: 00.26, \delta=+49: 53: 48.2$, with a positional uncertainty of $23^{\prime \prime}$ in each direction (estimated by bootstrapping the source catalog), which makes it consistent with the locations of the X-ray peak and the BCG at the $1 \sigma$ level.

Figure 5 displays the measured tangential reduced shear profile of $\mathrm{Cl} J 120958.9+495352$ as a function of the projected separation from the X-ray peak, combining measurements from all selected galaxies with $24<V_{606 \text {,aper }}<26$, as done in S16. Fitting these measurements within the radial range $300 \mathrm{kpc} \leq$ $r \leq 1.5 \mathrm{Mpc}$ assuming a model for a spherical NFW density profile according to Wright \& Brainerd (2000) and the 
mass-concentration relation from Diemer \& Kravtsov (2015), we constrain the cluster mass to $M_{500} / 10^{14} M_{\odot}=4.4_{-2.0}^{+2.2}$ (stat.) \pm 0.6 (sys.) and $M_{200} / 10^{14} M_{\odot}=6.5_{-2.9}^{+3.0}$ (stat.) \pm 0.8 (sys.).

Here we have corrected for a small expected bias of $-7 \%$ $(-8 \%)$ for $M_{500}\left(M_{200}\right)$ caused by the simplistic mass model, as estimated by S16 and further detailed in Applegate et al. (in prep.) using the analysis of simulated cluster weak lensing data. Differing from S16 we assume negligible miscentering for the bias correction, justified by the regular morphology of the cluster and precise estimate of the X-ray cluster center. The quoted statistical uncertainty includes shape noise, uncorrelated large-scale structure projections, and line-of-sight variations in the source redshift distribution, while the systematic error estimate takes shear calibration, redshift errors, and mass modeling uncertainties into account (see S16 for details). Here we have doubled the systematic mass modeling uncertainties used in S16 as we include somewhat smaller scales in the $\mathrm{fit}^{4}$. When restricting the radial range in the fit to the more conservative range $500 \mathrm{kpc} \leq r \leq 1.5 \mathrm{Mpc}$ from $\mathrm{S} 16$, the resulting constraints are $M_{500} / 10^{14} M_{\odot}=4.2_{-2.3}^{+2.6}$ (stat.) \pm 0.4 (sys.) and $M_{200} / 10^{14} M_{\odot}=6.3_{-3.4}^{+3.6}$ (stat.) \pm 0.6 (sys.) with smaller expected and corrected biases of $3 \%(5 \%)$ for $M_{500}\left(M_{200}\right)$ and smaller systematic uncertainties, but increased statistical errors.

For the comparison to the X-ray measurements we additionally require weak lensing mass estimates for an overdensity $\Delta=2500$. When assuming the Diemer \& Kravtsov (2015) mass-concentration relation and extrapolating the bias corrections ${ }^{5}$, the weak lensing mass constraints correspond to $M_{2500} / 10^{14} M_{\odot}=1.7_{-0.8}^{+0.9}$ (stat.) \pm 0.2 (sys.) when including measurements from scales $300 \mathrm{kpc} \leq r \leq 1.5 \mathrm{Mpc}$, and $M_{2500} / 10^{14} M_{\odot}=1.6_{-0.9}^{+1.0}$ (stat.) \pm 0.2 (sys.) when restricting the analysis to scales $500 \mathrm{kpc} \leq r \leq 1.5 \mathrm{Mpc}$.

We expect that our mass estimation procedure is unbiased within the quoted systematic uncertainties for a random population of massive clusters. For an individual cluster like the one studied here, deviations in the density profile from the assumed NFW profile with a concentration from the Diemer \& Kravtsov (2015) mass-concentration relation lead to additional scatter in the mass estimates. To estimate the order of magnitude of this effect we repeat the mass fits for scales $300 \mathrm{kpc} \leq r \leq 1.5 \mathrm{Mpc}$ using different concentrations. Based on simulations, Duffy et al. (2008) find that the scatter around the median concentration is approximately lognormal with $\sigma\left(\log _{10} c_{200}\right)=0.11$ for relaxed clusters. Approximately matching the expected $1 \sigma$ limits, fixed concentrations $c_{200}=3.0\left(c_{200}=5.0\right)$ change the best fit mass constraints for $M_{200}, M_{500}, M_{2500}$ by $+11 \%,+6 \%,-9 \%$ $(-11 \%,-5 \%,+11 \%)$ compared to the default analysis using the Diemer \& Kravtsov (2015) mass-concentration relation which yields a concentration of $c_{200}=3.7$ at the best fitting mass. These variations are negligible compared to the statistical uncertainties of the study presented here. It should be noted that this analysis

\footnotetext{
4 In the analysis of simulated data we find that the mass biases increase by factors of $\sim 1.6-2.3$ when changing from the default lower limit $>500 \mathrm{kpc}$ from S16 to $>300 \mathrm{kpc}$ as employed here. Following S16, we estimate the residual uncertainty of the bias correction as a relative factor of the bias value. Accordingly, the uncertainty increases by approximately a factor of two.

This is necessary given that the analysis from S16 as a function of $\log \Delta$ provides bias estimates for $\Delta=200$ and $\Delta=500$ only, as masses $M_{2500}$ are not available for the simulations used to derive the bias values. We do propagate the statistical uncertainty of this extrapolation, but note that it is negligible compared to the statistical uncertainty of the mass constraints for $\mathrm{Cl} J 120958.9+495352$.
}

Table 2. Global cluster properties between $0^{\prime}<R<1$ '.8.

\begin{tabular}{cc|c}
\hline \hline & Background-modeling & Background-subtraction \\
\hline$T[\mathrm{keV}]$ & $9.04_{-1.88}^{+1.38}$ & $8.84_{-0.71}^{+0.97}$ \\
$Z\left[Z_{\odot}\right]$ & $0.35_{-0.18}^{+0.20}$ & $0.46_{-0.17}^{+0.19}$ \\
norm $^{1}$ & $18.95_{-1.28}^{+1.32}$ & $19.09_{-0.73}^{+0.72}$ \\
\hline
\end{tabular}

Notes. ${ }^{(1)}$ Norm $=\frac{10^{-18}}{4 \pi\left[D_{\mathrm{A}}(1+z)\right]^{2}} \int n_{\mathrm{e}} n_{\mathrm{H}} \mathrm{d} V \mathrm{~cm}^{-5}$ with $D_{\mathrm{A}}$ being the angular diameter distance to the source.

assumes spherical cluster models, which can lead to extra scatter due to triaxiality when comparing to X-ray results.

In Fig. 4, the signal-to-noise ratio contours of the mass reconstruction appear to be slightly elliptical, extending towards the south-southwest, which is tentatively in agreement with the location of some apparent early-type cluster galaxies. To investigate whether this elliptical shape is actually significant, we estimate the shape of the mass peak using Source Extractor both for the actual mass reconstruction and the reconstructions originating from the bootstrapresampled catalogs. Using the Source Extractor estimates of the semi-major and semi-minor axes $a$ and $b$, as well as the position angle $\phi$ measured towards the north from west, we compute complex ellipticities $e=e_{1}+\mathrm{i} e_{2}=|e| \mathrm{e}^{2 \mathrm{i} \phi}$ with $|e|=(a-b) /(a+b)$, as employed in weak lensing notation (e.g., Bartelmann \& Schneider 2001). Using the dispersion of the estimates from the boostrapped samples as errors, our resulting estimate $e=(-0.05 \pm 0.18)+\mathrm{i}(-0.06 \pm 0.16)$ is consistent with a round mass distribution $(e=0)$. Hence, the apparent elliptical shape is not significant.

\subsection{XMM-Newton results}

\subsubsection{Global cluster properties}

The global properties for both methods of the treatment of the background are summarized in Table 2. The overall properties agree well between the two methods.

The rest-frame luminosity of the cluster in the $0.1-2.4 \mathrm{keV}$ band is $L_{\mathrm{X}}=\left(13.4_{-1.0}^{+1.2}\right) \times 10^{44} \mathrm{erg} / \mathrm{s}$ and $L_{\mathrm{X}}=\left(13.7_{-0.5}^{+0.5}\right) \times 10^{44} \mathrm{erg} / \mathrm{s}$, for background-modeling and background-subtraction method, respectively, estimated from the spectral fit. It is thus comparable to the most X-ray luminous MACS clusters, but at even higher redshift. These values are also in very good agreement with the findings by Buddendiek et al. (2015) after applying a K-correction.

\subsubsection{Temperature and density}

We compare the results for the two approaches of the background treatment for temperature and density profile. Figure 6 shows the temperature profile of $\mathrm{Cl} J 120958.9+495352$ for both approaches, and Table 3 gives the results.

Overall we see a very good agreement between the two different background methods. The temperature of the central bin is well constrained in both cases and both profiles show a good indication of a cool core. This makes $\mathrm{Cl} J 120958.9+495352$ one of only a few such objects known at high redshifts. The upper uncertainties in the outer two bins are large which is mainly related to the correlation between the parameters due to the PSF 


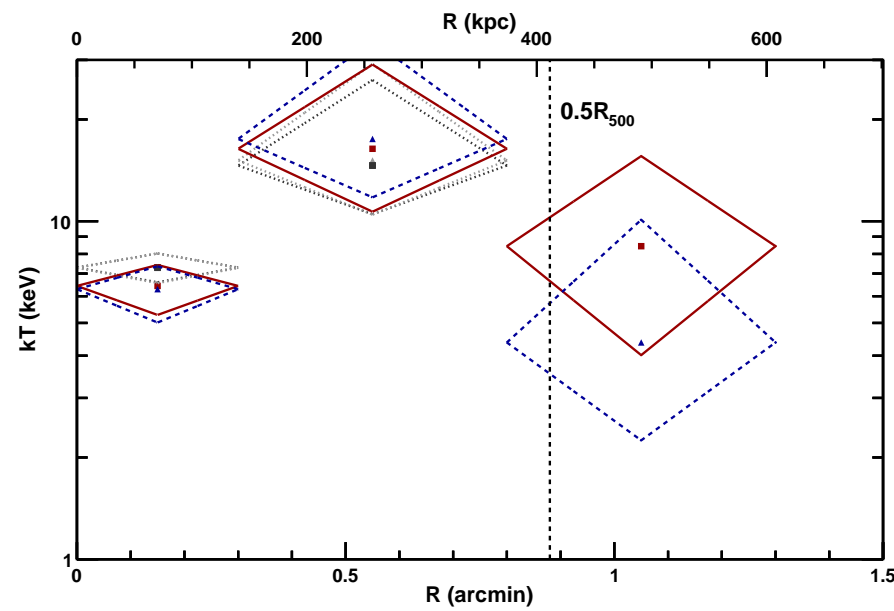

Fig. 6. Deprojected and PSF-corrected temperature profile of Cl J120958.9+495352. Red (dark gray) solid diamonds show the deprojected (projected) result using the background-subtraction method. Blue (light gray) dashed diamonds corresponds to the background-modeling method.

Table 3. Fit results for the three radial bins for both methods of background treatment.

\begin{tabular}{cccc}
\hline \hline & $0 \prime-0.3$ & $0.3-0.8$ & $0.8-1.3$ \\
\hline \multicolumn{4}{c}{ Background-modeling } \\
\hline$T[\mathrm{keV}]$ & $7.28_{-0.72}^{+0.75}$ & $15.13_{-4.67}^{+14.04}$ & $4.38_{-2.13}^{+5.72}$ \\
$Z\left[Z_{\odot}\right]$ & $0.25_{-0.14}^{+0.16}$ & \\
\cline { 2 - 4 } Norm $^{1}$ & $11.38_{-0.49}^{+0.58}$ & $5.40_{-0.46}^{+0.44}$ & $2.08_{-0.46}^{+0.86}$ \\
\hline & Background-subtraction \\
\hline$T[\mathrm{keV}]$ & $7.29_{-0.69}^{+0.74}$ & $14.61_{-4.13}^{+11.55}$ & $8.43_{-4.42}^{+7.15}$ \\
\cline { 2 - 4 }$Z\left[Z_{\odot}\right]$ & $0.32_{-0.15}^{+0.17}$ \\
\cline { 2 - 4 } Norm $^{1}$ & $11.24_{-0.51}^{+0.53}$ & $5.34_{-0.43}^{+0.44}$ & $1.82_{-0.28}^{+0.47}$ \\
\hline
\end{tabular}

Notes. The abundance is linked between all annuli. ${ }^{(1)}$ Norm $=$ $\frac{10^{-18}}{4 \pi\left[D_{\mathrm{A}}(1+z)\right]^{2}} \int n_{\mathrm{e}} n_{\mathrm{H}} \mathrm{d} V \mathrm{~cm}^{-5}$ with $D_{\mathrm{A}}$ being the angular diameter distance to the source.

correction and the limited statistics. Even if no PSF correction is applied, the cool core remains and the uncertainty of the second temperature decreases by a factor of $\sim 5$ and of the outermost temperature by a factor of $\sim 2$.

We determine the gas density profile using the PSF-corrected normalizations of the APEC model, which is defined as

$N_{i}=\frac{10^{-14}}{4 \pi D_{\mathrm{A}}^{2}(1+z)^{2}} \int_{V_{i}} n_{\mathrm{e}}(R) n_{\mathrm{H}}(R) \mathrm{d} V$,

where $i$ corresponds to the $i$ th annulus from the center and $D_{\mathrm{A}}$ is the angular diameter distance to the source. The volume along the line of sight $V_{i}$ is the corresponding cylindrical cut through a sphere with inner and outer radii of the $i$ th annulus. We adopt $n_{\mathrm{e}}=1.17 n_{\mathrm{H}}$. Due to the small extent of the cluster, there is only limited radial resolution. Therefore, we perform a simple deprojection method following Ettori et al. (2002).

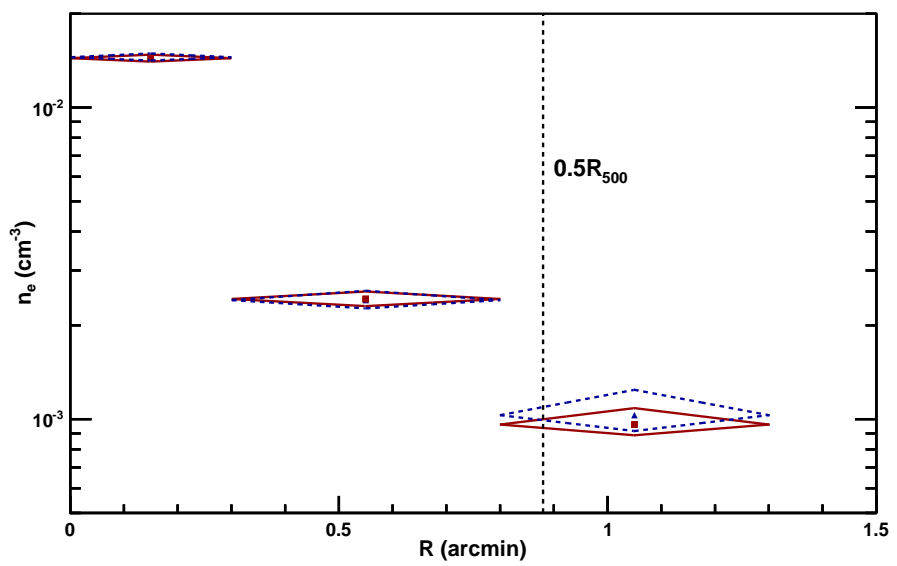

Fig. 7. Deprojected and PSF-corrected electron density profile of ClJ120958.9+495352. Red solid diamonds show the result using the background-subtraction method. Blue dashed diamonds correspond to the background-modeling method. The width of the diamonds corresponds to the radial bin size.

The emission integral $\left(E I_{i}\right)$ and temperature $\left(T_{i}\right)$ in ring $i$ are given by

$$
\begin{aligned}
E I_{i} & =\sum_{j=i}^{N} n_{\mathrm{e}} n_{\mathrm{H}} V_{i, j} \\
T_{i} & =\frac{\sum_{j=i}^{N} \epsilon_{j} V_{i, j} T_{j}}{\sum_{j=i}^{N} \epsilon_{j} V_{i, j}},
\end{aligned}
$$

with $V_{i, j}$ being the volume of the cylindrical cut corresponding to ring $i$ through spherical shell $j, n_{\mathrm{e}}$, and $n_{\mathrm{H}}$ the electron and proton density, and $\epsilon$ the emissivity. By subtracting the contribution of the overlying shells in each annulus, we determine the deprojected electron density profiles for both background treatment methods shown in Fig. 7. As for the temperature, the two density profiles agree very well showing that our background treatment works well in both cases.

As an additional test for the background-subtraction method we choose an even larger inner radius of the background region $\left(4^{\prime}-5^{\prime}\right)$ and repeat the analysis. We find only marginal differences and thus conclude that no significant cluster emission is present in the background-region.

As can be seen in Fig. 1, we detect a point source close to the center of the cluster. To investigate the impact of the point source, we increase the exclusion radius around this source by $50 \%$ and repeat the fit. Due to the lowered statistics, the uncertainties clearly increase but we find no significant impact compared to the nominal values.

\subsubsection{Gas mass fraction}

From the gas mass profile and the total mass $M_{\text {tot }}(<R)$ inside a given radius $R$, the gas mass fraction can be obtained:

$f_{\text {gas }}(<R)=\frac{M_{\text {gas }}(<R)}{M_{\text {tot }}(<R)}$.

We note that, given the limited $X M M$-Newton spatial resolution, a very robust determination of the total mass from the hydrostatic equation is difficult as this would require high spatial resolution measurements of the density and temperature profile. Therefore, we use the total mass based on our weak lensing HST estimates 
and the corresponding $R_{2500}$ (see Sect. 3.1). As a cross-check, we also determine the gas-mass fraction using the $L_{X}-M_{2500}$ relation obtained by Hoekstra (2007) for the total mass.

The HST results yield $M_{2500} / 10^{14} M_{\odot}=1.7_{-0.8}^{+0.9}$ (stat.) \pm 0.2 (sys.). For the estimation of $f_{\text {gas }}$, we include an additional $30 \%$ triaxiality/projection uncertainty and a $10 \%$ uncertainty from the mass-concentration relation on $M_{2500}$. From 10000 Monte Carlo (MC) realizations of $M_{2500}$, we estimate $R_{2500}=0.75_{-0.20}^{+0.13}$ and for each realization the gas mass within the corresponding $R_{2500}$, assuming a constant density in each shell. This yields $M_{\mathrm{gas}, 2500}=\left(1.64_{-0.67}^{+0.53}\right) \times 10^{13} M_{\odot}$ and $M_{\text {gas }, 2500}=\left(1.63_{-0.67}^{+0.53}\right) \times 10^{13} M_{\odot}$ for the background-subtraction and background-modeling method, respectively, which are in very good agreement. Combining these results, we estimate $f_{\text {gas }, 2500}=0.10_{-0.02}^{+0.03}$ for both methods. We note that through this procedure the given uncertainties on $M_{2500}, M_{\mathrm{gas}, 2500}$, and $R_{2500}$ are, on the one hand, correlated and, on the other hand, the assumption of constant density in each shell is only a rough approximation, which is why the uncertainty on $f_{\text {gas, } 2500}$ is lower than naively expected. A more general estimate is obtained by using a beta-model for the density profile and following the same procedure as described above. We fix the core radius to a typical value of $R_{c}=0.15 \times R_{500}$ and assume a slope of $\beta=2 / 3$ (as also used in, e.g., Pacaud et al. 2016), but including $15 \%$ scatter on the latter. $R_{500}$ is estimated from our HST results. This yields $f_{\text {gas }}=0.11_{-0.03}^{+0.06}$ for both background methods. Yet another approach is to estimate $f_{\text {gas }}$ and its uncertainties at a fixed radius (i.e., assuming that the true $R_{2500}$ is known), in which case the uncertainties on $M_{2500}$ and $M_{\text {gas }}$ are uncorrelated and directly propagate onto $f_{\text {gas }}$, which then yields $f_{\text {gas }}=0.11_{-0.05}^{+0.12}$. Here, we take the result using the beta-model as default.

Hoekstra (2007) estimated the $L_{\mathrm{X}}-M_{2500}$ relation for a galaxy cluster sample of $20 \mathrm{X}$-ray luminous objects at intermediate redshifts up to $z \sim 0.6$. They find a slope consistent with the one from Pratt et al. (2009), which is also used in the redshift evolution study of Reichert et al. (2011) and also consistent with the (inverted) slope of Maughan (2007) who assumed selfsimilar evolution. Using the relation from Hoekstra (2007) and assuming $30 \%$ intrinsic scatter, we find $M_{2500}=\left(1.31_{-0.29}^{+0.31}\right) \times$ $10^{14} M_{\odot}$ for the background-subtraction method and $M_{2500}=$ $\left(1.30_{-0.30}^{+0.32}\right) \times 10^{14} M_{\odot}$ for the background-modeling method and (using the corresponding $\left.R_{2500}\right) M_{\text {gas }, 2500}=\left(1.34_{-0.25}^{+0.27}\right) \times 10^{13} M_{\odot}$ and $M_{\text {gas,2500 }}=\left(1.33_{-0.30}^{+0.32}\right) \times 10^{13} M_{\odot}$, respectively. This yields $f_{\text {gas }, 2500}=0.10 \pm 0.02$ for both background methods, and is in very good agreement with our previous findings using the weak lensing mass.

\subsection{Cooling time}

To estimate the cooling time, we further reduced the size of the central region to 0.2 corresponding to $\sim 100 \mathrm{kpc}$ and performed the same PSF correction and deprojection method as described above. The cooling time is given by (Hudson et al. 2010)

$t_{\text {cool }}=\frac{3\left(n_{\mathrm{e}}+n_{\mathrm{i}}\right) k_{\mathrm{B}} T}{2 n_{\mathrm{e}} n_{\mathrm{H}} \Lambda(T, Z)}$,

where $n_{\mathrm{i}}$ is the ion density and $\Lambda(T, Z)$ the cooling function. Within $100 \mathrm{kpc}$ we find $n_{\mathrm{e}}=\left(2.09_{-0.08}^{+0.10}\right) \times 10^{-2} \mathrm{~cm}^{-3}$ and $T=4.0_{-1.5}^{+1.3} \mathrm{keV}$. This yields a short cooling time for $\mathrm{Cl} J 120958.9+495352$ within $100 \mathrm{kpc}$ of $t_{\mathrm{cool}}=2.8 \pm 0.5 \mathrm{Gyr}$ for the background subtraction method and $t_{\text {cool }}=2.9 \pm$ $0.4 \mathrm{Gyr}$ for the background modeling method. Hudson et al. (2010) studied the cool cores for a local sample of 64 clusters within $0.4 \% R_{500}$ with Chandra. According to their findings, ClJ120958.9+495352 belongs to the weak cool core clusters; however, it should be taken into account that the radius, in which they determine the cooling time, is much smaller than what is possible for $\mathrm{Cl} J 120958.9+495352$ and, presumably, within this radius the cooling time would be even lower, possibly resulting in a strong cool core classification.

\section{Discussion and conclusions}

Our results show that $\mathrm{Cl} J 120958.9+495352$, according to Planck Collaboration XVI (2015), belongs to the most luminous galaxy clusters known at $z \sim 0.9$. Compared to the total mass estimate from Buddendiek et al. (2015) of $M_{500}=(5.3 \pm 1.5) \times$ $10^{14} h_{70}^{-1} M_{\odot}$, we find a slightly lower value from our weak lensing analysis of $M_{500} / 10^{14} M_{\odot}=4.4_{-2.0}^{+2.2}$ (stat.) \pm 0.6 (sys.), which is, however, compatible within the uncertainties.

As discussed in, e.g., Sanderson et al. (2009) and Semler et al. (2012) there is a tight correlation between the dynamical state of the cluster and the presence and strength of a cool core. We find strong indications for the presence of a cool core, and the two different approaches for the background handling yield similar results which gives us confidence in our treatment of the background. The temperature profile shows a clear drop towards the center and the cooling time within $100 \mathrm{kpc}$ is short with $t_{\mathrm{cool}}=2.8 \pm 0.5 \mathrm{Gyr}$ and $t_{\mathrm{cool}}=2.9 \pm 0.4 \mathrm{Gyr}$ for the background-subtraction and background-modeling method, respectively. Another indicator for the morphological state is the offset between the BCG and the X-ray emission peak (see, e.g., Rossetti et al. 2016; Mahdavi et al. 2013; Hudson et al. 2010). Rossetti et al. (2016) define a relaxed cluster by an offset smaller than $0.02 R_{500}$. For $\mathrm{Cl} J 120958.9+495352$ the offset is about $2^{\prime \prime}(\sim 15 \mathrm{kpc})$ corresponding to $0.015 R_{500}$ (using the BCG position given in Buddendiek et al. 2015, see also Fig. 4), which is another indication for the relaxed nature of the system. Our HST weak lensing study also shows that the mass reconstruction peak is compatible with the BCG position and the X-ray peak within $1 \sigma$. As investigated in Sect. 3.1, the apparent elliptical shape of the lensing mass reconstruction is not significant. Hence, the results are consistent with a round mass distribution.

In a bottom-up scenario for structure formation, massive cool core systems should be extremely rare at high redshifts. Their gas mass fractions should not depend on the cosmological model. However, the apparent evolution varies for different assumed cosmologies. Previous measurements from Allen et al. (2008) and Mantz et al. (2014) show that their data are in good agreement with the standard cosmological model, showing a flat behavior of $f_{\text {gas }}$ with redshift. However, these data only contain a few objects at very high redshifts. Therefore, clusters like Cl J120958.9+495352 are valuable objects for cosmology.

We obtain a gas mass fraction of $f_{\text {gas }, 2500}=0.11_{-0.03}^{+0.06}$, which is consistent with the result from Allen et al. (2008) for their full cluster sample and also consistent with the assumed $\Lambda \mathrm{CDM}$ cosmology $\left(\Omega_{\mathrm{m}}=0.3, h=0.7\right)$. We performed several tests, i.e., we used an $L_{\mathrm{X}}-M_{2500}$ scaling relation for the total mass and tested the assumption of constant density in each shell, to verify this result and find very good agreement. Mantz et al. (2014) measured the gas mass fraction in an annulus from $0.8 R_{2500}<R<$ $1.2 R_{2500}$ excluding the core of the clusters to minimize gas depletion uncertainties and intrinsic scatter in the inner part. They 
find typical $f_{\text {gas }}$ values between $0.10-0.12$ and are thus consistent with our findings and Allen et al. (2008).

Reichert et al. (2011) studied the evolution of cluster scaling relations up to redshift 1.5 . They use the relations from Pratt et al. (2009) for the local clusters and obtain a biascorrected evolution factor. Testing this $L_{\mathrm{X}}-T$ scaling relation with our estimated global gas temperature yields a luminosity that is about $40 \%$ smaller than our measured value. This result is, at least partially, expected due to the presence of a cool core. However, the uncertainties solely due to the uncertainties of the slope and normalization of the scaling relation (assuming they are uncorrelated) are already large $(\gtrsim 40 \%)$.

The cluster $\mathrm{ClJ} 120958.9+495352$ is interesting not only with respect to cosmology, but also to its astrophysics. At redshift 0.9 the time span for this massive object to form a cool core is very short. As $X M M-N e w t o n$ is not able to fully resolve the core structure, we aim for higher spatial resolution data in a future project to robustly determine the X-ray hydrostatic mass and to perform a detailed study of the core properties.

Acknowledgements. This work is based on joint observations made with the NASA/ESA Hubble Space Telescope, using imaging data from program 13493 (PI: Schrabback) and XMM-Newton data (IDs 0722530101 and 0722530201), as well as WHT data (ID W14AN004, PI: Hoekstra). S.T. and T.S. acknowledge support from the German Federal Ministry of Economics and Technology (BMWi) provided through DLR under projects 50 OR 1210, 50 OR 1308, 50 OR 1407, and 50 OR 1610. S.T. and T.H.R. acknowledge support by the German Research Association (DFG) through grant RE 1462/6 and the Transregio 33 "The Dark Universe" sub-project B18. S.T. also acknowledges support from the Bonn-Cologne Graduate School of Physics and Astronomy. L.L. acknowledges support from the Chandra X-ray Observatory grant GO3-14130B and from the Chandra X-ray Center through NASA contract NAS8-03060. Support for Program number GO-13493 was provided by NASA through a grant from the Space Telescope Science Institute, which is operated by the Association of Universities for Research in Astronomy, Incorporated, under NASA contract NAS5-26555.

\section{References}

Allen, S. W., Rapetti, D. A., Schmidt, R. W., et al. 2008, MNRAS, 383, 879 Allen, S. W., Evrard, A. E., \& Mantz, A. B. 2011, ARA\&A, 49, 409

Asplund, M., Grevesse, N., Sauval, A. J., \& Scott, P. 2009, ARA\&A, 47, 481 Babyk, I. 2014, Balt. Astron., 23, 93

Bartelmann, M., \& Schneider, P. 2001, Phys. Rep., 340, 291

Bertin, E., \& Arnouts, S. 1996, A\&AS, 117, 393

Bleem, L. E., Stalder, B., de Haan, T., et al. 2015, ApJS, 216, 27

Buddendiek, A., Schrabback, T., Greer, C. H., et al. 2015, MNRAS, 450, 4248

De Luca, A., \& Molendi, S. 2004, A\&A, 419, 837

Diemer, B., \& Kravtsov, A. V. 2015, ApJ, 799, 108
Duffy, A. R., Schaye, J., Kay, S. T., \& Dalla Vecchia, C. 2008, MNRAS, 390, L64

Ebeling, H., Barrett, E., Donovan, D., et al. 2007, ApJ, 661, L33

Ebeling, H., Edge, A. C., Mantz, A., et al. 2010, MNRAS, 407, 83

Ehlert, S., Allen, S. W., von der Linden, A., et al. 2011, MNRAS, 411, 1641

Erben, T., Van Waerbeke, L., Bertin, E., Mellier, Y., \& Schneider, P. 2001, A\&A, 366, 717

Erben, T., Schirmer, M., Dietrich, J. P., et al. 2005, Astron. Nachr., 326, 432

Ettori, S., Fabian, A. C., Allen, S. W., \& Johnstone, R. M. 2002, MNRAS, 331, 635

Hoekstra, H. 2007, MNRAS, 379, 317

Hoekstra, H., Franx, M., Kuijken, K., \& Squires, G. 1998, ApJ, 504, 636

Hoekstra, H., Bartelmann, M., Dahle, H., et al. 2013, Space Sci. Rev., 177, 75

Hudson, D. S., Mittal, R., Reiprich, T. H., et al. 2010, A\&A, 513, A37

Kaiser, N., Squires, G., \& Broadhurst, T. 1995, ApJ, 449, 460

Koekemoer, A. M., Fruchter, A. S., Hook, R. N., \& Hack, W. 2003, in HST Calibration Workshop: Hubble after the Installation of the ACS and the NICMOS Cooling System, eds. S. Arribas, A. Koekemoer, \& B. Whitmore, 337

Luppino, G. A., \& Kaiser, N. 1997, ApJ, 475, 20

Mahdavi, A., Hoekstra, H., Babul, A., et al. 2013, ApJ, 767, 116

Mantz, A. B., Allen, S. W., Morris, R. G., et al. 2014, MNRAS, 440, 2077

Massey, R., Schrabback, T., Cordes, O., et al. 2014, MNRAS, 439, 887

Maughan, B. J. 2007, ApJ, 668, 772

Maughan, B. J., Jones, L. R., Ebeling, H., \& Scharf, C. 2004a, MNRAS, 351, 1193

Maughan, B. J., Jones, L. R., Lumb, D., Ebeling, H., \& Gondoin, P. 2004b, MNRAS, 354, 1

McDonald, M., Allen, S. W., Bayliss, M., et al. 2017, ApJ, 843, 28

McInnes, R. N., Menanteau, F., Heavens, A. F., et al. 2009, MNRAS, 399, L84

Menanteau, F., Hughes, J. P., Sifón, C., et al. 2012, ApJ, 748, 7

Pacaud, F., Clerc, N., Giles, P. A., et al. 2016, A\&A, 592, A2

Planck Collaboration XXXII. 2015, A\&A, 581, A14

Pratt, G. W., Croston, J. H., Arnaud, M., \& Böhringer, H. 2009, A\&A, 498, 361

Reichert, A., Böhringer, H., Fassbender, R., \& Mühlegger, M. 2011, A\&A, 535, A4

Rossetti, M., Gastaldello, F., Ferioli, G., et al. 2016, MNRAS, 457, 4515

Sanderson, A. J. R., Edge, A. C., \& Smith, G. P. 2009, MNRAS, 398, 1698

Schirmer, M. 2013, ApJS, 209, 21

Schneider, P. 2006, in Gravitational Lensing: Strong, Weak \& Micro, Saas-Fee Advanced Course 33, Swiss Society for Astrophysics and Astronomy, eds. G. Meylan, P. Jetzer, \& P. North (Berlin: Springer-Verlag), 269

Schrabback, T., Erben, T., Simon, P., et al. 2007, A\&A, 468, 823

Schrabback, T., Hartlap, J., Joachimi, B., et al. 2010, A\&A, 516, A63

Schrabback, T., Applegate, D., Dietrich, J. P., et al. 2018, MNRAS, 474, 2635

SDSS Collaboration, Albareti, F. D., Allende Prieto, C., et al. 2017, ApJS, 233, 25

Semler, D. R., Šuhada, R., Aird, K. A., et al. 2012, ApJ, 761, 183

Siemiginowska, A., Burke, D. J., Aldcroft, T. L., et al. 2010, ApJ, 722, 102

Simon, P., Taylor, A. N., \& Hartlap, J. 2009, MNRAS, 399, 48

Skelton, R. E., Whitaker, K. E., Momcheva, I. G., et al. 2014, ApJS, 214, 24

White, S. D. M., Navarro, J. F., Evrard, A. E., \& Frenk, C. S. 1993, Nature, 366, 429

Willingale, R., Starling, R. L. C., Beardmore, A. P., Tanvir, N. R., \& O’Brien, P. T. 2013, MNRAS, 431, 394

Wright, C. O., \& Brainerd, T. G. 2000, ApJ, 534, 34 